\title{
Psychological factors associated with adherence to treatment in type 2 diabetes
}

A. Mhalla ${ }^{1}$, H. Mhalla ${ }^{2}$, C. Amrouche ${ }^{2}$, M. Jabeur ${ }^{1}$, F. Zaafrane ${ }^{1}$, L. Gaha ${ }^{1}$.

1- Research Laboratory "vulnerability to psychosis", Faculty of Medicine of Monastir, University of Monastir, Monastir, Tunisia.

2- Nutrition Institute, Tunis, Tunisia.

\section{Introduction :}

The relationship between adherence to antidiabetic treatment and psychological factors in particular depression and self-esteem has been widely established in the literature.

The objective of this study was to determine the psychological factors associated with adherence to treatment in type 2 diabetes.

Methods :

\section{CROSS-SECTIONLA STUDY}

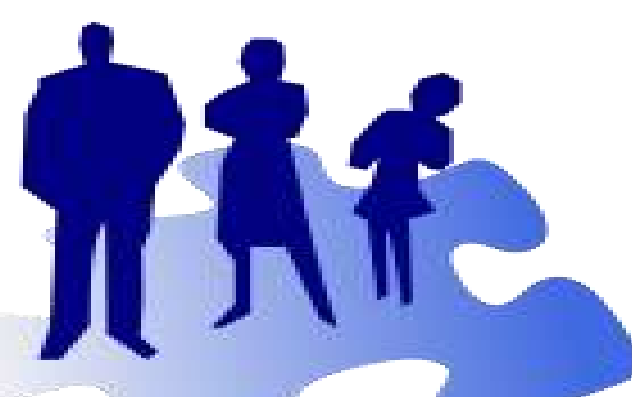

\section{Patients with type 2 diabetes attended at the Tunis Institute of Nutrition.}

\section{Psychometric evaluation :}

The Beck's scale for the assessment of depression

* The Morisky scale for the assessment of treatment adherence;

The Diabetes Treatment Satisfaction Questionnaire for the assessment of treatment satisfaction;

The Audit of Diabetes Dependent Quality of Life scale for the assessment of quality of life (ADDQoL).

Results :

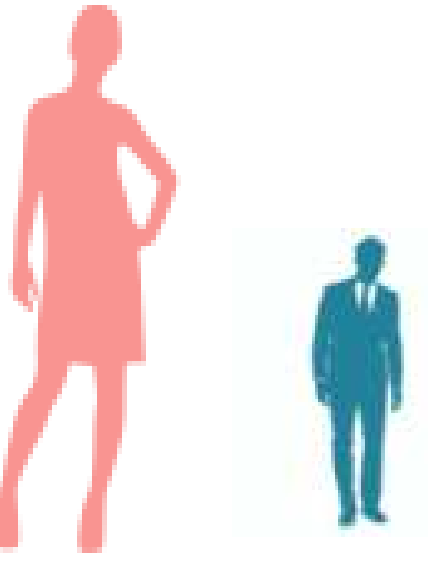

Mean age: $53.4 \pm 8.5$ years old sex-ratio $H / F: 0.7$

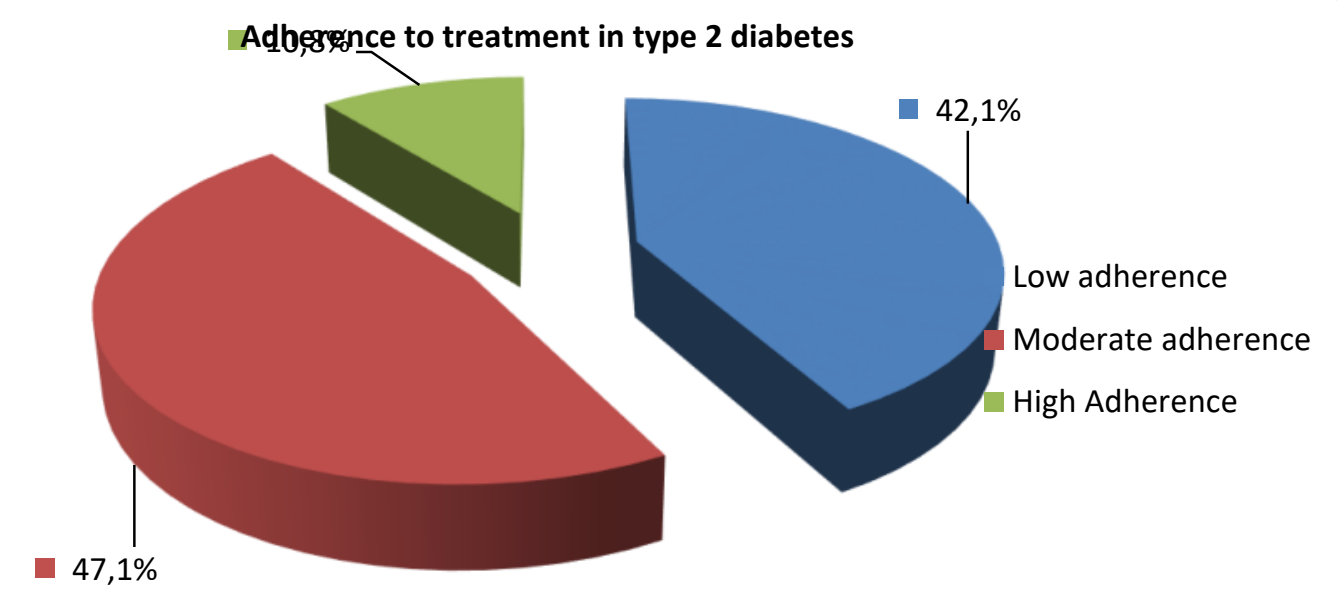

Tab1: factors associated with treatment adherence

\begin{tabular}{|l|c|}
\hline Age & Treatment adherence score \\
\hline BMI & $(r=-0,21 ; p=0,03)$ \\
\hline Glycémie à jeun & $(r=0,12 ; p=0,24)$ \\
\hline HBA1C & $\underline{(r=-0,26 ; p=0,009)}$ \\
\hline Beck scale score & $(r=-0,23 ; p=0,02)$ \\
\hline Self esteem score & $\underline{(r=-0,26 ; p=0,009)}$ \\
\hline Cyclothymic temperament score & $(r=0,20 ; p=0,04)$ \\
\hline Depressiv temperament score & $(r=-0,21 ; p=0,03)$ \\
\hline Irritable temperament score & $(r=-0,14 ; p=0,15)$ \\
\hline Hyperthymic temperament score & $(r=-0,28 ; p=0,005)$ \\
\hline Anxiuous temperament score & $(r=-0,03 ; p=0,72)$ \\
\hline Treatment satisfaction score & $(r=-0,19 ; p=0,05)$ \\
\hline
\end{tabular}

Conclusions :

Glycemic control and adherence to treatment in type 2 diabetes are associated with psychological factors such as depression, temperament and self esteem. Taking into account these factors in the management of diabetes is essential. 